REVUE DES PUBLICATIONS ÉTRANGËRES

\section{ELECTRICAL JOURNAL}

fUSION ELECTHLUE DU LATTON

II. St John dans Elect. J., (seplembre $19 \mathrm{~g}$ ), passe en revue les progrès accomplis dans la fusion électrique du laiton; il indique aussı les difficultie rencontrées et les caractéristiques des divers procédés acluellement utilisés.

\section{$* *$}

\section{ZEITSCHRIFT VEREINES DEUTSCHER INGENIEURE}

DÉVELOPPEMENT DE L INDUSTRE ĹLECTRO-CHIMIQUE ALLEMANDE

H. Goltschmidt dans Zills. Vereunes Deutsch. Ing. (13 et 20 septembre ig19) donne en deux articles des rensergnements intéressants sur le développement de l'industriè électro-chimique allemande, el la produclion des diverses fabrications, les procé dés employés, l'avenir de chaque industrie. L'Aluminum est préparé à partır de l'alumine et de la cryolithe dans des fours de fer revêtus de graphite, le procédé déjà ancien a été difficiloment perfectionné et la plupart des petites unutés employées, roo $H \mathrm{P}$. environ (200 en Amérique) ont de fortes pertes de courant dans les conducleurs et les connexions. La bauxite doit étre tratéc par la soude caustique et la proportion de silice contenue, détermine des pertes de soude, des essais ont été faits pendant la guerce en Allemagne pour préparer l'alumine à partur de l'argile, ils n'ont pas réussi, les usines nouvelles dépendront beaucoup de la France, riche en bauxile. Le procédé Serpek: préparation du nitrite d'Al. à partir de la bauxite et décomposition ensuite par la soude caustique pour obtenir l'alummate et l'ammoniarue, n'était pas réellement indusricl avant la guerre. Le Magnésium est produit par l'électrolyse de la carnallite fonduc; l'alliage dit "élcctron " consiste en $\mathrm{Mg} 95 \%$, Zn $5 \%$; il peut ètre laminé et embout mais les opérations demandent une grande ndresse. Le Sodium est ohtenu en électrolysant la soude fondue, l'électrolyse du sel marin est peu satisfaisante car il se forme un sous-chlorure. Carbure de Calcum La fabrication du rirbure de calcium est devenue très imporlante, et des usines completes onl été installées par diferses maisons. On emploie de grandes unités fonclionnant sous r 20 , ifo volls, 60 ooo ampères. Lounvier chargé du four est protégé contre les radiations calorifinues, mais les ouvriers ntilisés à la charge el à la conlée ne sont pas protígés; il y a un trou de coulée sous chacune des trois électrodes, capable d'ètre ouver par un are auxiliaire. les fumces des fours à carbure sonl trìs incommodantes, la précipitalion de ces fumées par le procédé Cottrell est seulement particlle Il $y$ a cing usines d'azotate de calcium : à Piesterilz, Chorzow, Trostberg (Bavè̀re), Waldshul (Bade) usine de la Lonza, Knappsack vers Cologne, L'alcool et l'ucade acétique ne peuvent se préparer que difficilement en Allemagne à partir du carbure de calcium.

Le Tungstène exporté on Angleterre avant la guerre élail fondu au creusel a partir du métal en poudre. La production électrothermuque du ferro-lungslène s'est récemment développée; mais los alliages à haute tencur (au-dessus de $75 \%$ de tungstòne) sonl rófractaires al ne peuvent être coulés et les alliages à teneur basse sont peu appréciés des métallurgistes. Fn ce qu concerne le ferochrome l'alliage a pourcentage faible et uniforme de carbone 0,5 à $0,7^{5} \mathrm{esl}$ difficilc à réaliser dans les fours électriques, rependani rette fabrication s'est développée pendant la guerre et pourra survivre si l'on peut obtenir des minerais de chrome. Un ferrochrome a io \% de $\mathrm{C}$ fat fait par Krupp avant la gnerre, Le Ferrosilicium est fabriqué également on Allemagne, le silicium lu-mêmo i Jaice en Bosnie, Ic graphite est obtenu à Ansug. L'acier flectrique eat produit dans les fours à radiation par l'are (Stassano, Héroult, Girod) of dans les fours à indurtion. Dạns les four's ITéroult le chanffage se fait par l'arc, le chauffage direct par to coulant est négligeable. Io four Nathusius est in four Girod aves un chanffage choctrique spécial du fonds. LC besoin de Ferro-manganèse dans le raffinage de l'acier peut etre réduit en ajoutant l'alliage fondu électriquement à l'acier, mais ll faul de la pratique. Le cuive et l'étaun des raffincries travaillant lo métal des cloches, n'est plus produit par suite du manque de mahère premiòre, il en sera de mème pour l'étain venant des usines de destannisation au moyen du chlore el lutilisation de ce dernier produit venant de l'électrolyse des alcalins deviendra difficile. Les procédés principaux d'électrolyse alcatone som reux de Griesheim el de la compagnie Aussig ; les bacs de cette dernière ne demandent pas des réparations fréquentes, mais peuvent sculement donner is chlorure de polassinm ; dans le chlorure de sodium on forme beancoup de chloralo soluble. La Griesheim Co ulilise des anodes de $\mathrm{Fe}^{3} \mathrm{O}^{4}$, obtenues par fusion de $\mathrm{Fe}^{2} \mathrm{O}^{3}$ a l'are électrique. Le procédé Billiler el la nouvelle cellule Gauss de la Badische Inilm und Sodafabrik, sont cmployés avec succès. Le Zinc très pur $(99,7$ à $99,9 \%)$ est cxtrail par électrolyse du sul. fate pur ou par distillation du zinc ordinaire dans les fours i radialion, le procédé scandinave vient doulte utilisé en Allema. gne ; il n'y a pas de progrès en ce qui ronccrne le traitement Électrothermique des minerais de zino

\section{$* *$ \\ ELECTRICIAN}

Drmanos

J. K. Catterson-Smilh dans Electrician (6 février 1920) considère dans la formule:

$\mathrm{K} \mathrm{W}=\mathrm{k} \mathrm{D}^{2} \mathrm{l}$ R $\mathrm{P} . \mathrm{M}$. donnant l'effet utile d'une dynamn, le coofficicnt k. Celui-ci est en général un cnsemble de facteur's renfermant les ampères tours par unité de longueur sur la circonférence. Liuteur estime que cette acception est insuffisanto et donne pour $\mathrm{k}$ la formule :

$$
\mathrm{k}=\mathrm{B}_{\mathrm{g}} \Delta\left(\frac{\pi^{2}}{60 \% \cdot \mathrm{r}_{\mathrm{s}} \mathrm{t} \cdot \mathrm{f}_{\mathrm{e}} \times 10^{-11}}\right)
$$

ou $B$ est la densilé du llux dans l'enlrofor, mesurée sur les faces - des pòles, $\Delta$ la densité de courant dans les spires, $\gamma$ le rapport do l'are polaire à la hauteur polaire, $r_{2}$ le rapport de la largen d'un silion interbobinaire à sa hauteur, t la profondeur d'un sillon, $\mathrm{f}_{\mathrm{e}}$ son facteur d'espace.

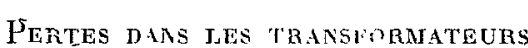

R. Kapp dans Electrcian ( 3 fóvrisp rgac) étudie les pertos dans les transformateurs Les pertes du fer se produisent lant que le transformateur est en relation avec la ligne, chargío ou non. Les pertes du cuive se produisent seulement fuand le transformateur est en charge, elles sont proportionnelles au carré de la charge. En cxprimant cela en $k w$-heures jar $k$ v.a. de caparite du transformateur placé, on'a : I) Perle du fer annuello = Pourcentage des pertes en fer $\times 8760 ; 2$ ) Pertes du cuivre annuello $=$ Pourcentage de la perte de cuivre en pleine charge $\times 8$ g $60 \times \mathrm{k}$. ou $\mathrm{k}$ est un farteur donné par :

\section{$\left(\Sigma\right.$ chargc $^{2} \times$ T'emps) [ (Taux d'efficacité du transformatcur $)^{2} \times \Sigma \cdot$ temps]}

I'n graphique montranl la relation entre les pertes du fer et celle dı cuivre est indiqué, et on pent l'utiliser pour déterminer rapidement quel est le rapport le plus lavoráble des perles pour un schéma déterminé. Un exemple est étudié dans lequel le transformaleur lo plus économique a un rendement en pleine charge, plus bas que toul autre; dans ce cas te meillem iransfornateur à employer a $0.38 \%$ pertes du fer, $1.14 \%$ pertes du cuivre, et $98.5 \%$ rendement a pleine charge. En complant 30 francs par k.v a., ce transformaleur serait (dans les conditions d'utilisation examinćes) beaucoup plus éconornique qu'un transformaleur conlant 2.5 fr. par k.v.a. avec pertes du fer el du cuivre à $0.65 \%$ et un rendement à pleine charge de $98.7 \%$. Dans un but de concurrence les fabricanls tendent a faire des transformateurs avec le

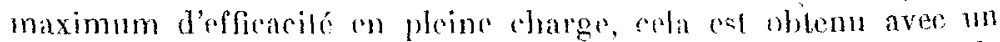
rapport des pertec de for anx pertes de cuivre égal à l'unité ; le prix supplémentaire d'un transformatcur dans lecquel il $y$ a de 
faibles pertes du fer el de fortes perles pour le cuivre, serait de 10 à $20 \%$. Dans-le cas considéré plus haut, le rapport le plus économique du fer au cuivre est environ $\mathrm{I} / 3$, mais il est entendu que ce rapport varic avec la courbe de charge; il peut se rapprocher de l'unitó dans lics petits transformateurs et devenir inférieur à $\mathrm{x} / 3$ dans les grands.' Des conditions de standardisation déterminent aussi les modèles, mais poúr avoir un maximum d'économie, l'acheteur doit spécifier que la somme des pertes du fer el d'unc fraction décrminée des pertes' du cuivre à pleine charge, doit être la plus fäible possible.

Cette fraction qui dépend de la courbe de charge, eśt $27 \%$ dans l'excmple choisi.

\section{INSTITUTE MINING}

ELONOMIE DU COMBUSTIBLF

J.A . Cork ot W. Hill dins Inst Mirning Eng., (Trans 5,7 août r gra) domnent un résumé des recherches ef des résultats obtenus au point de vue de la consommation du combustible dans dés usines de types variés. Cés úsines appartiennent à la « Midland Coal, Coke and Iron Co Ltd s).

L'appareillage fut établi de façon à employer les sous produits du régénéraleur, à récupérer les premiers produits ohtenus, enfin à utiliser le supplément de gaz pour obtenir la force électrique. Dans les ancicnnes usines, la consommation de la vapeur en comprenant les pertes dues à la radiation ou à d'autres causes variait de $16 \mathrm{~kg}$. o 7 à $30 \mathrm{~kg}$. 85 par cheval-heure, la vapeur était distribuéc sous une pression de $3 \mathrm{~kg}$. 6 à $7 \mathrm{~kg}$. 20 par $\mathrm{cm}^{2}$ et l'efficacité thermique atteignait en moyenne $2,26 \%$. La nouvellc usine, misc en fonctionnement en $\mathrm{I} g \mathrm{I} \mathrm{r}$, agrandie en $\mathrm{Ig} \mathrm{I} 3$, produisait depuis cotte dernière daté jusqu'en août $\mathrm{r}^{3} 4, .5$ millions de chevaux ; le facteur moyen de charge atteignait 0.727 , le rondement thermique $20, \mathrm{r} 3$ L'addition d'une autre machinc a gaz en IgI8 a amené le nombre d'appareils de l'usine au total de 4 machines à 8 cylindres de 600 chevanx chacune couplees directement avec des allernateurs triphasés de 550 volts, 35 périodes.

\section{ELECTRICIAN}

CONDUGteurs D'ALUMinium POUR LIGNes dE FORCE

A. Jarob dans Electrician (27 juin rgrg) résume d'abord les considérations relatives aux lignes de trânsport ì haute tension, puis en fäit l'application à l'emploi de conducteurs d'aluminium. La conductibilité de l'aluminium et dı cuivre, leur densité, la résislance à la traction etc., sont disculées ; le problème de la flèche des fils est envisagé ainsi que la nature des supports, bois ou acier. Les prix de revient relatifs du cuivre, de l'aluminium, de l'acier-aluminium on tant que conducteurs sont de même calculés ; enfin une étude rapide de quelques systèmes de transmission utilisant des câbles d'aluminium est donnée.

\section{ELECTRICAL WORLD}

RECONSTRUCTION DFS LIGNES DE TRANGPORT

P. Revneau et H. Scelve dans El. Word (3 janvier 1920) étudient les principes et les méthodes à suivrc dans la reconstruction d'une ligne de force. Coùt des améliorations, reconslruction, nouvelié posc, utilisation des vicux matériaux et autres problèmes sont ainsi cuvisagés.

\section{Soudure a L'ARO}

A. Hudson dans El: World (og novembre, 6 décembre igig) íludic li théorie de la soudurc à l'arc. Quand un arc éclate entre une électrode d'acier et une plaque d'acier, l'extrémité de l'électrode et unc partie de la plaque sont chauffées à une température Clevée, du métal est transporté de l'électrode à la. plaque. Qucllo est la cause de cette tránsmission ? L'autenr montre que le métal déposé l'est, en partic tout au moins, soins forme de particules très fines projetécs de l'électrode par. la dilatation de quelque vapeur, peut-être de CO. Les particules expulsées passent à travers l'arc trop rapidement pour se vaporiser et atteignent la placque seulement à l'état fhuide. Si elles atteignent le métal solide, elles ricochenl sur la surface ou s'aplatissent sans fusion, loutes causes de soudure pen solide. Si les particules atteignent metas liquide, elles le pénètrent et se solidifient avec la surface fonduc. Tout produit qui peut accroître le point de fusion de la surface d'une électrode doit, d'après celle théoric, améliorer les conditions sous lesquelles les particules sont projetées à partir de l'électrode. La plupart des recouvrements d'électrodes semblent satisfaire. ì cela par leur vaporisation et parfois étant donnée la rapidité de l'action, restent dans un état fluide sur les còtés de l'électrode.

Les électrodes rouillées font, en général, du meilleni travail que les électrodés bion nettes, car le poinl de fusion de FoO est plus élevé que celui de l'acicr. L'électrode idéale devrait avoir une écaille externe à point de fusion élevéc, forméc, par exemple, de tungstène, entourant un intérieur de proinl de fusion plus bas formé de constituanls capables de donner assez de vapeurs pour projeter constamment le métal.

\section{$* *$ \\ ENGINEER}

Soudure a I'ARe DANS LA COVSTRUCTHON des Navities

O. Kjellberg dans Engineer (3 octobre et 7 novembre igrg) étudic quelques propriétés de pièces métalliques soudécs à l'arc. Des essais faits sur des pièces miquement préparées à la soudure électrique ont montró une ténarité de $4.000 \mathrm{kgs} \mathrm{par} \mathrm{cm}^{2}$, le module d'élasticité est à peu près le même que pour des piècrs forgées ou laminées.

Le détail de ces essais est donné. En outre, uno statistique est faitc des réparations , effectuées au moyen de l'are à Gothenburg depuis rgo4; 2068 chandières, 700 hélices el axcs, $: 43$ gouvernails, 58 étraves et étambots ont été réparés. Les soudures défectucuses n'ont pas dépassé - 0 \% \% dans le cas des chaudières, pour les axes o $3 \%$, pour les gouvernails $0.5 \%$; en ce qui concerne les étraves et étambots une seule fut défcctueuse et encore pas à la place de soudure. Iin grand nombre de réparations ont aussi été cffectuées sur des chaudières et des vaisscaux classés dans le Lloyd's Régister. Enfin les exigences de la guerre ont déterminé des progrès considérables dans l'emploi de la soudure électrique pour la construction de nouveanx vaisseaux La quantité de sol1dure à faire dans un navire est énorme, foo mètres par jour. Lc rapport entre la sondure ct le rivetage prul s'exprimer aniss 600 mètres de soudure contre 7.000 rivets. La possibilité d'obtenir des sondures plus fortes a fait nâ̂tre des idées nouvelles en ce qui concerne la construction des navires ; par exemple, construire un vaisseau par sections de $r \mathrm{~m}$. 80 , la plupart disposées transversalement tandis que seules les pièces de l'avant sont placces longitudinalement; c'est la méthode la plus simple Une journce de huit heures suffirait à la pose, la soudure et l'érertion d'me section entière de $\mathrm{x} \mathrm{m}$. $8 \mathrm{n}$; on placcrait en même temps la machinerie de sorte que, dès que la coque serait prête, le vaisseau pourrait prendre la mer. On estime à $20 \%$ le poids de matiere ćconomisée par un tel procédé. Isa vitesse de soudure fa plus grande est $\mathrm{r} 40 \mathrm{~cm}^{3}$ de dépôt par heure, la moyenne, go. Ainsi un hon soudeur peut achever $4 \mathrm{~m}$. So de souditre par heure, si fos plaques à souder sont hien asaujelties ; une movenne de 3 mètros est la plus raisonnable. L'énergie électrique utilisée par unitó de volume de saudure est la même quelle que soit l'épaisscur.

\section{ELECTRICAL WORLD}

Brts D'rsolatetirs

F. Kallevang dans El. World (r3 décombre rgrg) decril un: méthode permettant de déceler les isolateurs défectume ; il note entre autres, le fait que des isolateurs restés plusienrs années en magasin sont détériorés Cette détérioration peut être due à des forces mécaniques on aux essais électriques. Les isolatours placés près des chemins de fer se hrusent facilement, pent-cttre par action de $\mathrm{SO}^{2}$ qui attaque le ciment et détermine la cristallisation on encore parce que noircis par la fumée ils penvent arçuérir des températures trop élevées. Le haut pourcentage de fêlures dans 
les isolateurs élevés est lû au fait que ces isolateurs sont soumis à des changements trop brusques de température. Les arbres qui sont an voisinage des lignes ou meme les surptombent sont une source fréquenle de dommages.

\section{ELECTRICAL REVIEW (CHICAGo)}

Lamies électriques

G. Shepherd dans El. Rev. Chicago (20 décembre rgig) donne des renscignements sur la durée des lampes utilisées dans le Pare Lincoln et cinq autres pares plus petits de Chicago ainsi que sur les boulevards adjacents. Pour l'éclairage extérieur 1,761 lampes de 400 bongies 15 ampères, sont placées; pour l'éclairage des monuments, des bords des pièces d'eau 300 lampes allant à un maximum de 1 ooo watts ; l'éclairage inlérieur est assuré par 6500 lampes de valeurs varićes. La méthode permettant de tenir le compte de la duréc d'une lampe est décrite et le nombre d'hcures moyen d'éclarage par mois ainsi que le total des mois. Les lampes brûlant toute la nuit (4.000 heures par in), la moilic de la muil (2.200 heures par an) ou allernativement tonte la nuit ol ane moilié de nuit (3.roo heures par ann) ne domment pas fividemment le môme nombre d'heures d'ćclairag̣e, mais brùlent presque tontes a la mème date On peut en conchure que la duréc de la lampe dépend heaucoup plus du nombre d'inferruptions el de fermetures auquel clle est soumse que du nombre d'hemres d'enploi.

\section{INFORMATIONS}

\section{Le Zinc Métal.}

Nous extrayous de l'Industrie chimique les renseignements suirelatif's à la situation du marché français en cé qui concerne le zinc :

Après celle du fer et celle de l'aluminium, la métallurgic du zinc est l'industric métallurgique la plus importante en France. Votre industrie du zinc n'a pas toujours compris tous les stades de la fabrication. Pendanl longtemps, nous nous bornàmes à faire passer au laminoir le zinc importé. Puis nous eréâmes des usines pour traiter nos minerais et l'cxtraction iudigène ayant subi de rogs à rgı 3 une diminution do pròs de moitié,nous fümes, sur me échelle de plus en plus large, tributaires des minerais élrangers.

Cette sujétion pèse sur notre industrie du zinc ainsi que la pénurie de loulle (il fant 4 tomes de houille pour fabriquer une Lonne de zinc) et la rareté de la main-d'œuvre. Mais ce sont là des obstacles qui ne s'opposent pas seulement an développement de la métallurgie du zinc en France, mais à celui de toutes les industriés métallurgiques françaises.

\section{Production française du Zinc.}

La production française du métal étail en rgr 3 de 67.890 tonnes gui ae décomposaient comme suit d'après les slatistiques de l'Induslrie muciale.

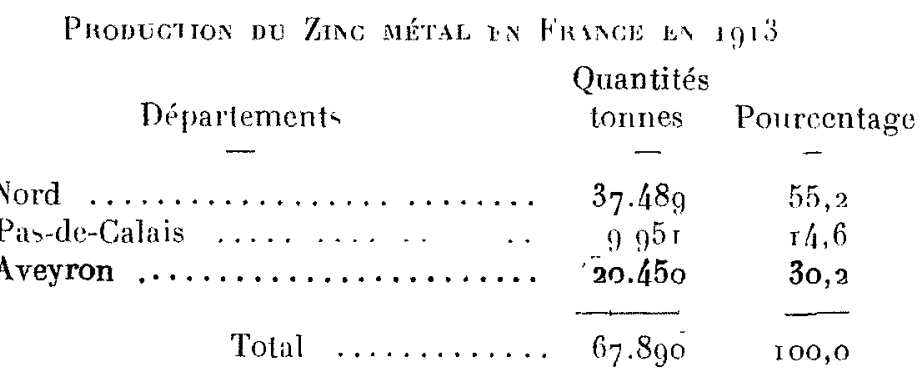

La presque totalití des usines sont siluŕes sur le charbon Viviez dans l'Aveyron, Noyelles-Godault dans le Pas-de-Calajs Iuby, Mortagne et Saint-Amand dans le Nord. Il convient d'ajouter Creil dans l'Oise.
Sur ces 67.890 tomnes, 12.750 seulentent provenaient des mine$t$ ais nationaux.

Depuis le débul du siè ln, notre produrtion de zinc a été croissunte, passant de 37.400 tomnes en 1913 à 48.000 lommes en 1908 el à 67.890 lonnes en 1913 .

Bien qu'en progròs la production frangaise du zane est très faible, si on la compare a la production mondiale quit, à la veslle de la gucre, alleignait un million de tomnes.

\section{Production-mondiale du Zinc.}

La mélallurgic du zine est dominée par la production de tres pays : les Ftats-Unis, l'Allémagne et la Belgique qui, ì utux trois, onl fabriqué en 1933,800 ooo tomnes, soil les $4 / 5$ de la produrtion mondiale. En 1880 , les Etats-lnis ne fournissaient pas ro \% de la production tolale, dès 18 yo, ils donnaient près de $18 \%$, en rgoo plus de $23 \%$ ot en rgr3 plus du r/3 de ce que produit le monde entier.

L'Allemagne ot is Belgique ont vu leur importance diminned censiblement : produivanl $45 \%$ en 1880 , l'Nllemagne, des $190 \%$, ne domnait que $33 \%$ ef en r $913,8,3 \%$. La Belgique, pays d'orrfrine de la mólallurge dit zinc, autrefois riche on minerais, de mande la quasi-lotalité de ses blendes el do ses calamines à l'étranger el voit ainsi sa production baiseer, par rapport à la producfion mondiale do $27 \%$ en 1880 à $19,8 \%$ cn 1913 .

La production mondiate de zine métal, qui élatt de 347.400 tonnes en 1890 , siest élevéc ì 472.000 tonnes en rgoo, à 817.000 - lunnes en rgro el à un million de lonnes en igr3. Comment " répartissait ce million de tonnes ? Le tableau suivant, emprunlí al Rapport Général sur l'Indusirie française, pubjié par le Ministire du Commerce, en donne une idée aussi cxacle que poseilif.

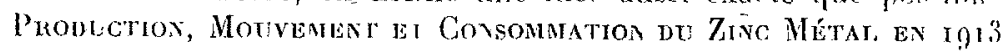

\begin{tabular}{|c|c|c|c|c|}
\hline Pays & $\begin{array}{c}\text { Production } \\
\text { tonnes } \\
-\end{array}$ & $\begin{array}{c}\text { Iniporlation } \\
\text { tonnes } \\
-\end{array}$ & $\begin{array}{c}\text { Exportation } \\
\text { tonnes } \\
-\end{array}$ & $\begin{array}{c}\text { Consommatio } \\
\text { tonnes }\end{array}$ \\
\hline Elats-linis & 320.300 & 5.500 & 12500 & $3 \times 3.500$ \\
\hline$\ldots \ldots$ & 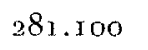 & 5 & 105. & 23 \\
\hline elgique $\ldots \ldots \ldots \ldots$ & 197. & 20. & $\mathrm{~T} 4 \mathrm{I}$. & 76.400 \\
\hline$\ldots \ldots \ldots \ldots$ & 67.890 & $39 \cdot 99^{5}$ & $29 \cdot 732$ & 78.153 \\
\hline$\ldots \ldots \ldots$ & $9.46 \mathrm{r}$ & 一 & 一 & 5.900 \\
\hline -Bretagne $\ldots .$. & $59 r / 46$ & $147.300^{\circ}$ & $16.8 \mathrm{I} 8$ & $\times 94.600$ \\
\hline$\ldots \ldots$ & 4.300 & - & - & $4.000(1)$ \\
\hline$\ldots \ldots$ & - & $12.1 / 18$ & 1.566 & $10.900\left(^{1}\right)$ \\
\hline che-Hongrie .... & 217 & - & - & $\left.40400^{1}\right)$ \\
\hline$\ldots$ & 92 & - & - & - \\
\hline & 7.61 & $25.7^{\circ}$ & - & 33.300 \\
\hline ralje $\ldots$ & 3.791 & - & -1 & 20.900 \\
\hline
\end{tabular}

Production

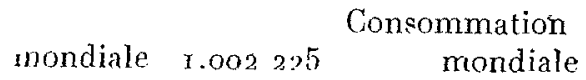

т.010.053

Ainsi la France ne vicnt qu'au quatrième rang des producteurs '2t des consommateurs de zinc. En rgr 3 . sa production ayant atteint 67.890 tonnes et sa consommation 78153 tonnes, son déficit a donc été de ro 263 tonnes.

\section{Le Commerce exIérieur du Zinc en France.}

lie dóficit, comment a-t-il été comblé ? D'une façon beaucoup plus complexe qu'on pourrait se l'imaginer tout d'abord, puisque pour y parvenir nous avons importé, en $1913,39.495$ tonnes de zinc et exporté 29.732 tonnes.

Nos importations ct nos exportations de zinc se décomposent (n) zine en saumons, barres et plaques, en zinc laminé et cn limailles et débris de vieux ouvrages.

En r.93, la Belgique nous envoyait $84 \%$ de nos exportations en zinc. brul (29.465 tonnes sur un total de 35.172 tonnes) Ja presque totalité du métal laminé( ${ }^{2} 57$ I tonnes sur $\mathrm{x} .594$ tonnes) et la moitié du zine en limailles et débris ( 1.800 tonnes sur 3.230 tonnos) Majs nous étions fortement exportateurs de zinc on fruilles, ce qui atteste la vitalité remarquable de notre industrie de transformation du mótal. En laminó. nous expédiions on Belgique un

(1) Fstimations 
poids double de celui que nous recevions ( 3.452 tonnes contre r. $57 \mathrm{I}$ ) et nous étions les pourvoyeur's de l'Italie (2.23 r tonnes), de la Suisse ( 995 tonnes), de nos colonies (1.20r tonnes). br tinc wa saumons, barres et plaques, nous exportions en Igr3, I8 291 tonnes, dont 9.964 à destination de la Belgique, 5.767 tonnes à destination de l'Angleterre et $x .392$ tonnes à destination de l'Italue.

\section{L.a Métallurgie du Zinc el la Guerre.}

Du lait de la guerre, la France a été privée de la plus grande partie de ses usines de zinc. Des usines nouvelles el des augmentations de fonderies existantes ont permis de compenser une partie de ces pertes. Les usines nouvelles sont celles de Rochcfort à la Cic Royale Asturienne d'Auby (capacité de production : 6.00o tonnes) et de Salindres à la Société des zines purs (capacité de production : r.zoo tonnes). D'autre part, les usines de Viviez ont acnru leurs moyens de production de 24.000 tonnes par an.

En présence des besoins considérables créés par la guerre, les autres pays. né sont pas restés infactils. La production des EtatsLnis est devenue formidable, passant de 1913 à 1917 de 32.000 fomnes à $7 \mathbf{1} 2.000$ tonnes $\mathrm{La}$ production du Canada, où des installations électrolyliques ont été créées, a atteint 14.200 tonnes en 1917 . De son còté, la production anglaise est passée de 59.000 tonnes à 120.000 tonnes. En Australie ont été montées deux ou trois usines électrolyliques, pouvant produire 45.000 tonnes par an. $\Lambda u$ point de vue tcchnique, des progrès très intéressants ont élé effectués, principalement aux Etats-Unis, dans les installations uhlisant l'électrolyse.

\section{La Métallurgie du Zinc el ta Paix.}

Nous avons rapıdement décrit la situation de lindustric du zinc en France pendant la guerre el au cours de la guerre. Quclles sont ses perspectives d'avenir?

Le lapport Général du Ministère du Commerce sur l'Industrie française en trace ainsi les grandes lignes. Nous nouveaux moyens de production permettent une augmentation de 30 .ooo tonnes. En plus, ọ peut attendre des nouvelles méthodes élcctrolytiques une excension de la production du zinc en France de 20.000 tonnes. djoutons ces 50.000 tonnes à la production de jgl 3 et notre proiluction sera près d'alteindre r 20.000 tonnes.

Ces 120.000 tomnes de zinc métal exigent un approvisionn'ment en minerai de 260.000 tonnes que la France peut sassurer de la façon suivante:

Production de la métropole en $1913 \ldots \ldots \ldots$.... 50.000 tonnes "des colonics frangaises en $1913 \ldots$. , 50 ooo"

" des miner françaises appartenant ì des sociétés françaises. ...........

Augmentation de production au cours de la guerre $\ldots \ldots \ldots \ldots \ldots \ldots \ldots \ldots$.

Tolal ........ 260 ooo lonnes

La consommation du zine qui état en France $7^{8.153}$ tonnes en 1913, atteindra sans exagération 130.000 tonnes. Le déficil de production sera donc de 19.000 tonnes. Il apparaît bien qu'on puisse le combler par une augmentat on de production-de nos usinc et l'emploi de la méthode électrolytique.

Quant aux approvisionnements en minerais correspondant à ce supplément de production, ils pourraient etre facilement obtenus par le dévoloppement de nos usines pyrénéennes.

\section{Ecole Supérieure d'Électricité.}

\section{Promotion XXVI. - r $1919-1920$}

\section{EXAMENS DE SORTIE}

Le Jury d'examens de sortie, présidé par M. Berlhelot, membre de l'Institut el de l'Académie de Médecine, Professeur à l'Université de Paris, dans la séance du 29 juillet 1920 , a accordé le diplôme d'Ingénieur-Electricien aux élèves dont les noms suivent :

Officiers délégués par le Ministère de la Guerre :

MM. le Capitaine du Génic............... Laqueille. - $\quad$ - $\ldots \ldots \ldots \ldots \ldots \ldots \ldots$ Godbille.

le Capitaine d'Artillerie.............. Allain.

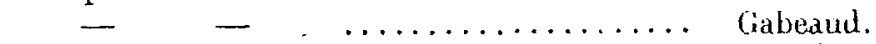

le Capitaine d'lnfanterie.............. d'Ornano.

le Capitaine d'Arthllerie............... Samuel.

le Capitaine du Service Automobile........ Brissot.

Officters el Ingénteurs délégués par le Ministère de la Marine. MM. le Lieutenant de Vaisseau............. Collin.

$$
\begin{aligned}
& \text { - } \quad \text { - } \quad \ldots \ldots \ldots \ldots \ldots \ldots \ldots \text { Collinet. } \\
& -\quad-\quad \ldots \ldots \ldots \ldots \ldots \ldots \ldots \ldots \ldots \text { Bonneau. }
\end{aligned}
$$

le Héçanicien princıpal de $\mathrm{I}^{10}$ classe........ Taillefer.

l'Ingénieur de $\mathrm{r}^{\text {re }}$ classe du Génic $\mathrm{N}^{\text {no }} \ldots \ldots$... Mestraud.

l'Ingénieur de ${ }^{\text {re }}$ classe d'Artil. nav...... Jamel.

Ingénieùrs stagiaures du Géné Rural délégués par le Ministère de l'Agriculture.

MM. Calvet et Olivié.

'Ingénieurs et Elèves-Ingénieurs des T'élégraphes.

WM. Lange.

Le Corbeiller.

\section{Elèves réguliers}

MM.

I Ozanne.

$2 \mathrm{M}^{\text {ile }}$ Bourgolghon.

3 Richemond.

4 Bondot.

5 Mingez.

6 Ileidmann.

7 Devilud.

8 Lemenand.

9 Clavier.

Io Breil.

I Girard.

12 Acquié.

13 Sigrist.

I4 Bourdel.

I5 Poizat.

i6 Marville.

i7 Dormont.

18 . Bès.

19 Nabères.

20 Lilnglois.

2 I Carrié.

22 Tauzin.

23 Murtin.

24 Montindon.

25 Bellan.

25 Gastine.

27 Lob-Lévyt.

28 Rougeulle.

29 Wiallut.

3o Michel.

3o Rives:

3. Pasquilini.

33 Geoffroy.

34 Depriester.

35 Préjet.

36 Datin.

37 Ujorljévitsh.

37 Verdelot.

39 Risch.

40 Roussel.

41 Doat.

4. Blondin.

42 Sonquière.

44 Bourgeois.

45 Mcynieux.

46 Pagès.

47 Poncy-Mounou.

\section{Vitérans:}

Mi

Laurent (1918-19).
48 Arpin.

18 Puig.

50 Bastide.

5o Vitu.

5. Holtzapffel.

53 Moniol.

54 Kocchlin.

54 Lucquel.

50 Laroche.

56 Perier.

58 Gires.

59 Carchereux.

io Boissé.

6I Abrial.

li II IIofer.

(i3 Demetz.

63 Denormandie.

65 Demanche.

(iti Maillard.

i60 Petitjean.

(ii) Raynaud.

by Pégourier.

7o Mathieu.

7 I Dumora.

72. Soulié.

73 Pelit.

73 Olliver.

-5 Moinet.

76 Franc.

$77 \mathrm{Bel}$.

78 Libourel.

7) Rothatcin.

So Rivlère.

BI Jac.

82 Coudé du Foresto.

83 Fourcade.

$8 / 4$ Denizet.

85 Doignon.

85 Maeder.

87 Moiroud.

8. Liailler.

si) Magnez,

jo Sovillart.

9I Baratte.

92 Bouis.

n' Milet.

gh Juget.

Mily (Ig13-14):

Nandin (IgI $8-19)$.
$9^{5}$ Gentlen Mey de C. gi Astoin.

gi Peyroulet.

gs Chavanier.

99 Lefève.

too Chrélien.

roo Pradon.

102 Lerond.

Io3 Dusotorl.

rol Bouteleux.

rof Pionnice.

10 i Vaulrin.

Io7 Murphy.

108 Mattepserre.

109 Montition.

$110 \mathrm{M}^{\text {Ho }}$ Schnerb.

III Bosio.

iI Baroche.

Ir 3 Schweitzer.

$114 \mathrm{De}$ Chambure.

I 5 Chambiard.

I 6 Cavaillé.

II Guerber.

I 8 Souplet.

I 9 Artous.

I 19 Durand (Ch.)

I'I Choix.

y.3 Du Bourg do Boz

I23 Sidi.

r.4 Murtinoff.

i?5 Lcroux.

I 9 Seguin.

I 26 Stein.

ig8 Hirtz.

I 29 Lyon.

I3o Gruat.

131 Delhaye.

I3, Corbeil.

I33 Ilerzensthin.

134 Prévost.

135 Mounicr.

30 Lhuillier.

I37 Krasner.

I38 Licheleau.

139 Vangier.

s(i) Poudiere. 


\section{Union des Syndicats d'Ingénieurs.}

La Commission intersyndıcale du Statul militaire de l'ingénieur composée de MM.-Albert Ranc el Sorde, ingénieurs-chimistes ; Guéry, Raoux, de Richemont, Rieunier, ingénięurs-électriciens ; Bourgeois, Chol, Pelit, ingénieurs-mécaniciens ; a remis au bureau de l'Union des Syndicats d'Ingénieurs le rapport de $M$. de Richemont sur la mobilisation technique et industrielle. Ce document, qui envisage la création d'un organisme supérieur réglant cette mobilisation ainsi que toules les mesures a prendre pour assurer le passage rapide de la siluation de paix ì la situation de guerre des usines el autres établissements scientifiques et techniques, a été renvoyé à l'examen d'urgence des Conseils d'administration des divers syndicats.

\section{LÉGISLATION}

ste par ordre chronologıque des arrétés el décrets se rapporlant aux industries de la houlie blanche ct de la houble noire (juillel, aoul, septembre, I920).

Arrêté fixant les prix de vonte aux consommateurs des agglomérés (briquetles el boulets ovoldes) en provenance des mines affiliées à la chambre de compensation des houillères du Centre (arrondissement minéralogique de Cłermont-Ferrand (Journal Officiel du 25 juillet 1920 , page xo643).

Décrel nommant des professeurs titulaires à l'école nationale supérieurc des mines (Journal Officiel du 27 juillet 1920, page $1(1780)$.

Décrel nommant des professeurs titulaires à l'écolè nationale supérieure des mines (Journal Officıel du 27 juillet r920, page 10780).

Circulaire pour les importateurs consommateurs et importateurs revendeurs de charbon (Journal Officiel du 27 juillet I 920 , page 10780 ).

Décret déclarant d'utilité publique l'établissement d'une canalisation dite " pipe line " servant au transporl du Havre à Par’s des huiles de pétrole (Journal Officiel du 28 juillet 1920 , page ro800).

Décret fixan! le nombre des membres dı Comité consultatif des charbons (Ioumal Officiel du 28 juillet r92o, page 10828).

Arrêtés nommant des membres du Comité consultatif des charbons (Journal Officiel du 29 juillet I 920 , page 10828).

Décret déclarant d'utulité publique létablissement d'une cana: lisation dite "pipe line " servant au transport du Havre à Paris des huiles de pélrole (errata) (Journal Officiel du 2 août íg2o, page rio3o).

Arreté fixant les prix de vente des charbons de provenance britannique (Journal Officıel du 2 aontt 1920 , page rıo3i).

trêté fixant les prix de vente des charbons de provenance, amóricaine (Journal Officiel du 2 août 1920 , page 1 Io33).

Arrcté fixant le prix de vente aux-consommateurs des agglomérés en provenance des houillères d'Ahun (Journal Officiel du 2 aon̂l Ig2o, page xio33).

Arrêté relatif à la péréquation des charbons à gaz (Journal Officiel du 3 aon̂t 1920 , page п 1065$)$.

Arrêté fixant les pris $d$ vente des charbons britanniques (rrata) (Journal Officiel du 7 août r920, page $\mathrm{x} 3389$ ).

Arrêté relatif à la péréquation des charbons à gaz (erratum) (Journal Officiel du 7 août I920; page I 390 ).

Décret portant règlement d'administrátion publique pour l'exécution de la loi du if octobre I 19 , en ce qui concerne la forme et la procédure d'inetruction des demandes de concessions d'usines hydrauliques sur les cours d'eau et les lacs, l'instruction des projets et leur approbation, ainsi que la forme des enquêtes relatives à l'élablissemcul des serviludes (Journal Offuciel du '8 aoùt x 920 , page $1 \times 468)$.

Décret portant ì̀glement d'administration publique pour l'exécution de la lor du 6 oclobre igrg, et lixant la forme et la procédure d'instiuction des denandes d'aulorisalion d'usines hydrauliques sur les cours d'eau et les lacs, l'instruction des projets el leur approbation (Journal Offlcelel du 8 aoùl 1920, page $\mathrm{r} / 47 \mathrm{r}$ ).

Décret portant règlement d'administration. publique pour lapplication de l'article 28 , paragraphe $2^{\circ}$, de la loi du 16 octoilre 19 rg et approuvant le modèle de règlement d'eau pour les entreprises autorisées sur les cours d'eau domaniaux el non domaniaux (Journal Officiel du 8 aoùt 1920 , page $1 \times 473$ ).

Note aux consommateurs de charbons (Journal Officiel du 14 août ig2o, page 11889 ).

Décret" portant règlement d'administration publique pour l'application de la loi du 23 avril 1919 sur la journée de huit heures dans les entreprises de produclion et de distribution d'énergic électrique de la région parisienne (Journal Offucıel du a septembre 1920, page 12853).

Arrêté fixant le nombre des représentants des exploitants de lourbe appelís à siéger au sein de la commission de la tourbe (Journal Officiel du a seplembre 1920, page 12784).

Arrêté nommant un mêmbre de la Commission de la tourbe (Journal Officiel du 2 septembre I'g2o, page 12784 ).

\section{BIBLIOGRAPHIE SCIENTIFIQUE}

Mesures Electrotechniques, par Albert Turpatn, Professcur à la Faculté des Sciences de Poitiers, chargé du Cours d'Electricité industrielle.

Un volume $16 \times 25$ de 183 pages, avec 105 figures. Prix (majoration comprise), 20 francs: - Dunod, éditeưr, Paris. - En vente à la librairie Rey, Grenoble.

Suivaul la méthode inaugurée, il y a quinze ans déjà, par l'àuteur - et qui fit le succès de ses ourrages de Pliysique expérimenlale - les mesures' pralıques d'électricité industrielle nécessăres à l'étudiant,' à l'ingénieur, à l'électricien, sont ici systématiquement exposées.

La réunion, dans des tahleaux d'expérience judicieusement disposés, des données, permet de dégager immédiatement lè résullaf cherché, tant en ce qui concerne la technique expérimentale des courants continus que des courants alternalifs.

Les Compteurs d'electric lté, par M.-R. Fichtex, 'ingénieus I. E. N. - Un vólume $16 \times 25$ de Iv-223 pages, avec 155 figures. Prix (májoration comprise), 24 francs. - Dunod, édileur, Paris. - En vente à la librairic Rey, Grenob́le.

L'ouvrage de M. Fichter explique sans détails superflus, mais avec tous renscignements utiles, le fonctionnement des divers types de compteurs, employés tant dans la pralique courante que paur les tarifications modernes les plus spéciales. Conçu dans un esprit éminemment pratique, ell mème $1 \mathrm{cmps}$ que crilique, dit W. Mauduit dans sa préface, il constitue un guide qui sera biẹn rite reconnu indispensable à lingénieur et au monteur, auxquels il fournira, nettement et rapidement, la solution de tous les problèmes posés par l'utilisation des compteurs : mode de branchement, étälonnage ef réglage, recherche des défauts ou accidenls.

Le Gérant: P. LEGENDRE

Anc. Etabts Legevdre, 14, rue Bellecordière, Lyon. - J Batalllard, Directeur. 